\title{
Strength-based and fracture-based approaches in the analysis of fibre debonding
}

\author{
C. K. Y. LEUNG, V. C. LI* \\ Department of Civil Engineering, Massachusetts Institute of Technology, Cambridge, Massachusetts, \\ USA
}

The debonding behaviour of fibres strongly affects the properties of fibre-reinforced composites. In the literature, two different approaches to the fibre debonding problem have been developed. In strength-based approaches [1-4], interfacial debonding is assumed to occur once an interfacial strength is reached. In fracture-based approaches [5-9], the debonded interfacial zone is regarded as a tunnel crack which grows in size once an interfacial toughness is overcome at the crack tip. In this communication, the conditions for validity of these two different approaches will be discussed with respect to different possible stress distributions along the interface. A method to determine which approach is appropriate for a given composite system will be suggested.

In most debonding analysis, it has been assumed that there is a sharp boundary between the debonded and undebonded regions of the interface. However, in reality, between the elastic undebonded zone and the frictional debonded zone, there may exist a transition zone (see Fig. 1) where breakdown of material takes place. If the transition zone is large (in comparison to fibre length), there is a smooth transition of interfacial stress from the undebonded zone to the debonded zone. On the other hand, if the transition zone is very small, there is an abrupt change between the two zones and a singular stress field will be present. In general, for large and small transition zone sizes and large and small $\tau_{2} / \tau_{\mathrm{i}}$ (Note: Here, $\tau_{\mathrm{s}}$ is the interfacial strength while $\tau_{\mathrm{i}}$ is the interfacial friction), four different cases can be distinguished. The various cases (I to IV) are shown schematically in Fig. 1. Cases I and II are for small $\tau_{\mathrm{s}} / \tau_{\mathrm{i}}$. For case $\mathrm{I}$, where the transition zone is large, there is no singularity in the stress field and a strength-based approach is appropriate. Since the difference between interfacial strength and interfacial friction is not significant, a single parameter $\tau_{\mathrm{i}}$ can be used to characterize both the transition zone and the frictional zone. For case II, where the transition zone is small, a stress singularity exists and a fracture-based approach should be more appropriate. However, if the interfacial toughness (usually denoted by a critical interfacial energy release rate $G_{\mathrm{c}}$ ) is low, once the debonded zone has extended beyond several fibre diameters, the contribution of frictional shear stress becomes significant compared with the contribution of elastic stresses in the undebonded zone. If one is interested in global composite behaviour (such as the relation between applied stress and fibre displacement) which is insensitive to the inaccuracy of stresses at local points, the use of a strength-based theory with an approximate stress field (such as one obtained from the shear lag analysis) may provide a good approximation. However, if the interfacial toughness is high, debonding is always dominated by the singular stress field and a fracture-based approach has to be used. Cases III and IV are for large $\tau_{s} / \tau_{i}$. For case III, where there is a large transition zone, the change of stress with slip in the transition zone (or the slip-weakening relation) can significantly affect interfacial behaviour. In this case, to study the debonding behaviour, linear elastic fracture mechanics will not be applicable because of the invalidity of the small scale yielding requirement. However, approaches similar to Barenblatt's [10] or Hillerborg's [11] for mode I "cohesive crack" or Li's [12] for mode II shear rupture, with the cohesive stresses given by the slip-weakening relation, can be employed. For case IV, where the transition zone is small, debonding behaviour is governed by the singular stress field and a fracture-based theory based on a single fracture parameter (such as the critical energy release rate) is appropriate. In general, a singularity may be maintained even when the transition zone is large such as in coarse grain alumina or certain fibre reinforced ceramics. For this case, a nonlinear fracture analysis similar to that for case III but which can consider both the crack tip singularity and the slip-weakening in the crack wake has to be carried out for the interfacial crack.

Experimental observations in support of the above arguments are available. For a silicon carbide reinforced lithium alumino-silicate system where there is negligible chemical bond between the fibre and matrix (i.e., $\tau_{s} / \tau_{\mathrm{i}}=1$ ), Marshall and Oliver [13] have shown that a frictional sliding analysis (which is equivalent to a strength-based analysis with the effect of elastic shear stress transfer neglected) gave good agreement with experimental measurement of the applied load versus fibre displacement curve. This observation is in agreement with the above discussions which suggest that the strength-based approach is valid for low $\tau_{s} / \tau_{i}$ (case I or case II). On the other hand, Piggott [7] shows that for a glass reinforced polyester resin system where $\tau_{\mathrm{i}}$ is greatly reduced by Poisson's contraction of fibre near the loaded end (i.e., $\tau_{\mathrm{s}} / \tau_{\mathrm{i}} \gg 1$ ), a strength-based analysis cannot provide a

* Now at the Advanced Civil Engineering Materials Research Laboratory, Department of Civil Engineering, University of Michigan, Ann Arbor, MI 48109-2125, USA. 


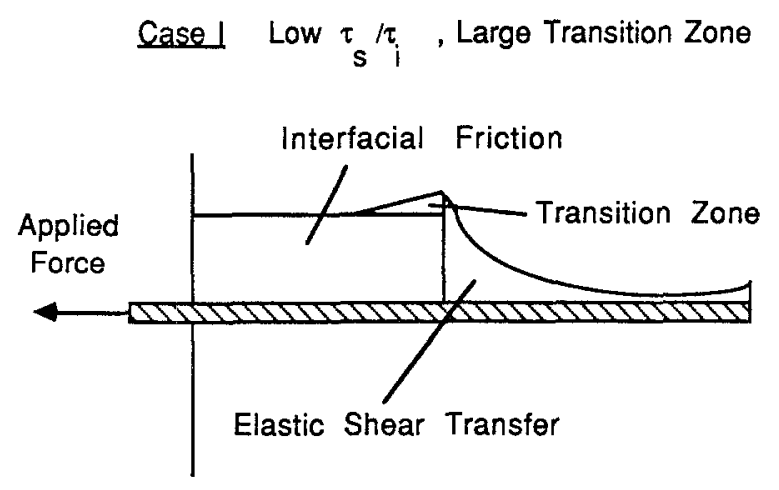

Case III High $\tau_{s} / \tau_{i}$, Large Transition Zone

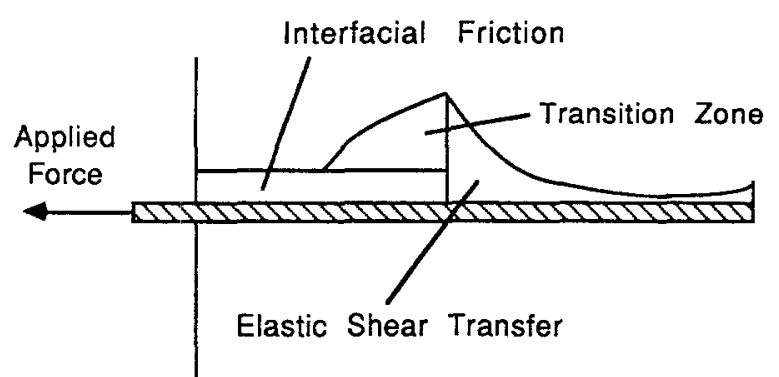

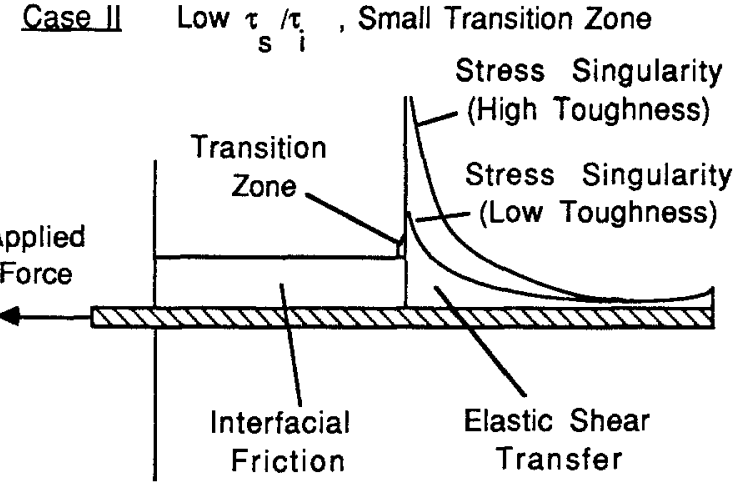

Case IV High $\tau_{s} / \tau_{i}$, Small Transition Zone

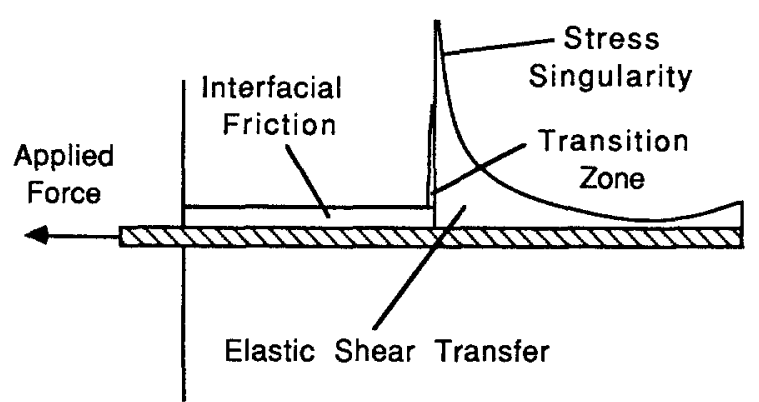

Figure $l$ Various possible distributions of interfacial shear stress for large and small transition zones and high and low ratios of interfacial strength to interfacial friction.

good fit to experimental data of maximum load against fibre length while a fracture-based theory with a single fracture parameter is able to explain the trend of experimental data. This composite system is thus a plausible example of case IV.

The above discussions suggest that a transition value of $\tau_{s} / \tau_{i}$ for the validity of strength-based or fracture-based approach should exist. Such a transition value should also depend on $G_{\mathrm{c}}$. Therefore, theoretically, if both $\tau_{s} / \tau_{\mathrm{i}}$ and $G_{\mathrm{c}}$ are measured for a certain composite system, one can decide which approach is appropriate. However, a technique which can separately measure $\tau_{\mathrm{s}} / \tau_{\mathrm{i}}$ and $G_{\mathrm{c}}$ is currently not available. To interpret data from current interfacial testing techniques, debonding has to be first assumed to be either strength-governed or fracturegoverned. The result is not very meaningful because it is not known whether the assumed situation is indeed the case. Thus, for available techniques, it is not possible to tell from one single set of test data which approach should be used. However, if data are available from tests on specimens with different parameters (such as different fibre radii or different fibre volume fractions), one can tell from the trend of experimental data whether debonding is strengthgoverned or fracture-governed. To illustrate the point, let us consider the interpretation of a fibre pull-out test.

A typical pull-out test result is shown in Fig. 2. The same data can be interpreted by the strength-based or the fracture-based approach. If a strength-based approach is employed (i.e., debonding is assumed to be strength-governed) with the stress distribution in the bonded zone obtained by an approximate shear lag analysis, it can be shown [14] that:

$$
\begin{aligned}
P_{\text {peak }} / P_{\text {postpeak }}= & 1+\left\{\left[\left(\tau_{\mathrm{s}} / \tau_{\mathrm{i}}\right)^{2}-\left(\tau_{\mathrm{s}} / \tau_{\mathrm{i}}\right)\right]^{1 / 2}\right. \\
& \left.-\cosh ^{-1}\left(\tau_{\mathrm{s}} / \tau_{\mathrm{i}}\right)^{1 / 2}\right\} /\left(\rho L / r_{\mathrm{f}}\right)
\end{aligned}
$$

where $P_{\text {peak }}$ and $P_{\text {postpeak }}$ are as shown in Fig. 2, $L$ the embedded fibre length, $r_{\mathrm{f}}$ the fibre radius and $\rho$ a parameter depending on moduli of fibre and matrix as well as fibre volume fraction in the pull-out specimen.

On the other hand, if debonding is assumed to be fracture-governed, the pull-out test data can also be interpreted with a fracture-based approach. Following an approach developed by Gao et al. [9], which considers the effect of interfacial friction on specimen compliance in the computation of energy release rate, and using the same approximate stresses from the shear lag analysis in [14], it can be shown that:

$$
\begin{gathered}
P_{\text {peak }} / P_{\text {postpeak }}=1 \\
+\left\{X^{1 / 2}-\cosh ^{-1}\left[0.5+(0.25+X)^{1 / 2}\right]^{1 / 2}\right\} /\left(\rho L / r_{\mathrm{f}}\right)
\end{gathered}
$$

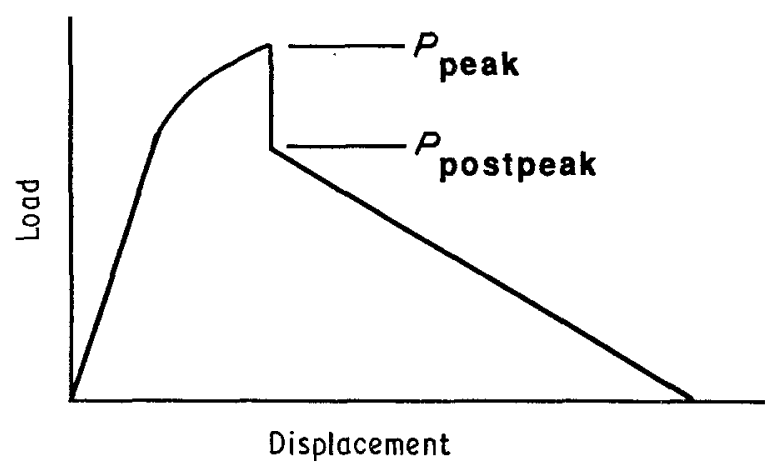

Figure 2 A typical pull-out test resuit. 
where $X=\left(E_{\mathrm{f}} G_{\mathrm{c}} \rho^{2}\right) /\left(\tau_{\mathrm{i}}^{2} r_{\mathrm{f}}\right)$ and $E_{\mathrm{f}}$ is the fibre modulus.

Depending on which approach is used, $\tau_{\mathrm{s}}$ or $G_{\mathrm{c}}$ can be obtained from $P_{\text {peak }} / P_{\text {postpeak }}$. (Note: $\tau_{\mathrm{i}}$ is obtained by dividing $P_{\text {posipeak }}$ by the total interfacial area $2 \pi r_{\mathrm{f}} L$.) Therefore, in the case where debonding is indeed fracture-governed (and should be characterized by $G_{c}$ and $\tau_{\mathrm{i}}$ ), we will obtain an equivalent $\tau_{\mathrm{s}}$ which is of no significant physical meaning if a strength-based approach is used. Thus, meaningful result is not guaranteed if only a single set of test data is available.

Replacement of $\left[0.5+(0.25+\mathrm{X})^{1 / 2}\right]$ in equation (2) and $\tau_{\mathrm{s}} / \tau_{\mathrm{i}}$ in equation (1) by $Y$ will give rise to the same equation as follows:

$$
\begin{aligned}
P_{\text {peak }} / P_{\text {posipeak }}= & 1+\left\{\left(\mathrm{Y}^{2}-\mathrm{Y}\right)^{1 / 2}\right. \\
& \left.-\cosh ^{-1} \mathrm{Y}^{1 / 2}\right\} /\left(\rho L / r_{\mathrm{f}}\right)
\end{aligned}
$$

To see whether debonding is strength-governed or fracture-governed for a certain composite system, one can carry out pull-out tests with different fibre radii or different fibre volume fractions (and hence different values of $\rho$ ). $Y$ is then obtained from equation (3). If $Y$ is a constant with respect to $r_{f}$ or $\rho$, debonding is strength-governed and $Y$ is equal to $\tau_{\mathrm{s}} / \tau_{\mathrm{i}}$ (compare equation (3) with equation (1)). If $Y$ varies with $r_{\mathrm{r}}$ and $\rho$ according to $Y=\left[0.5+(0.25+\mathrm{X})^{1 / 2}\right]$, debonding is governed by a single fracture parameter $G_{\mathrm{c}}$ (and $\tau_{\mathrm{i}}$ ). If $Y$ varies with $r_{\mathrm{f}}$ and $\rho$ in other manners, it is possible that the debonding process is governed by more complex mechanisms such as the presence of a large slip-weakening zone or the presence of both a crack tip singularity and a large slip-weakening zone.

\section{References}

1. L. B. GRESZCZUK, in ASTM STP 452 (1969) p. 42

2. A. TAKAKU and R. G. C. ARRIDGE, J. Phys. D: Appl. Phys. 6 (1973) 2038.

3. P. LAWRENCE, J. Mater, Sci. 7 (1972) 1.

4. V. S. GOPALARATNAM and S. P. SHAH, ASCE $J$. Eng. Mech. 113 (1987) 635.

5. C. GURNeY and J. HUNT, Proc. Roy. Soc. Lond. A299 (1967) 508.

6. H. STANG and S. P. SHAH, J. Mater. Sci. 21 (1986) 953.

7. M. R. PIGGOTt, Comp. Sci. Tech. 30 (1987) 295.

8. J. K. MORRISON, S. P. SHAH and Y. S. JENQ, ASCE J. Eng. Mech. 114 (1988) 277.

9. Y. GAO, Y. W. MAl and B. COTTERELL, J. Appl. Math. Phys. (ZAMP) 39 (1988) 550.

10. G. I. BARENBLATT, in "Advances in Applied Mechanics," Vol. 7, edited by H. L. Dryden and T. von Karman (Academic Press, New York, 1962) p. 56.

11. A. HILLERBORG, in "Fracture Mechanics of Concrete," edited by F. H. Wittmann (Elsevier Science, B. V. Amsterdam, 1983) p. 223.

12. V. C. LI, in "Fracture Mechanics of Rock," edited by B. Atkinson (Academic Press, London, 1987) p. 351.

13. D. B. MARSHALL and W. C. OLIVER, J. Amer. Ceram. Soc. 70 (1987) 542.

14. C. K. LEUNG and V. C. LI, "Applications of a TwoWay Debonding Theory to Short Fibre Composites," submitted to Composites (1989).

Received 3 January

and accepted 5 February 1990 